\section{(2) \\ BRAZIILIAN JOURNAL \\ OF MEDICAL AND BIOLOGICAL RESEARCH}

www.bjournal.com.br
ISSN 0100-879X

Volume 45 (7) 565-680

July 2012

CLINICAL INVESTIGATION

Braz J Med Biol Res, July 2012, Volume 45(7) 637-643

doi: 10.1590/S0100-879X2012007500065

Emergence of clonal complex 5 (CC5) methicillin-resistant Staphylococcus aureus (MRSA) isolates susceptible to trimethoprim-sulfamethoxazole in a Brazilian hospital

M.M. Teixeira, M.C. Araújo, M.C. Silva-Carvalho, C.O. Beltrame, C.C.H.B. Oliveira, A.M.S. Figueiredo and A.G. Oliveira

The Brazilian Journal of Medical and Biological Research is partially financed by

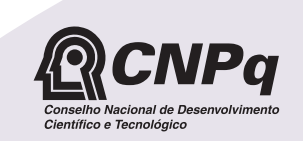

da Ciência e Tecnologia

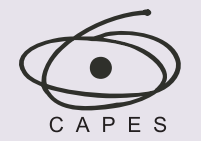

Ministério da Educação
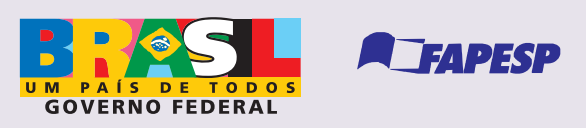

Institutional Sponsors
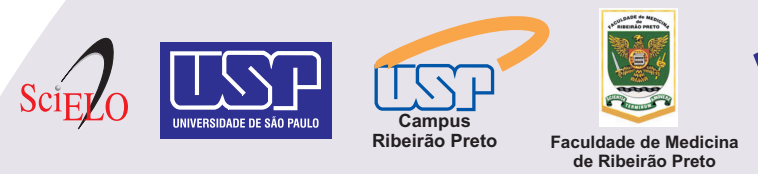

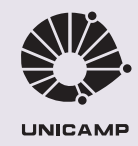

shimadzu

UNICAMP

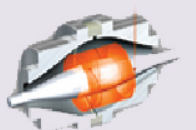




\title{
Emergence of clonal complex 5 (CC5) methicillin-resistant Staphylococcus aureus (MRSA) isolates susceptible to trimethoprim- sulfamethoxazole in a Brazilian hospital
}

\author{
M.M. Teixeira ${ }^{1}$, M.C. Araújo1 ${ }^{1}$ M.C. Silva-Carvalho², C.O. Beltrame², \\ C.C.H.B. Oliveira ${ }^{3}$, A.M.S. Figueiredo ${ }^{2 *}$ and A.G. Oliveira ${ }^{1 *}$ \\ 1 Instituto de Ciências Biológicas e Naturais, Universidade Federal do Triângulo Mineiro, Uberaba, MG, Brasil \\ 2Departamento de Microbiologia Médica, Instituto de Microbiologia Paulo de Góes, \\ Universidade Federal do Rio de Janeiro, Rio de Janeiro, RJ, Brasil \\ ${ }^{3}$ Departamento de Clínica Médica, Universidade Federal do Triângulo Mineiro, Uberaba, MG, Brasil
}

\begin{abstract}
In this study, genotyping techniques including staphylococcal chromosomal cassette mec (SCCmec) typing, pulsed-field gel electrophoresis (PFGE), multilocus sequence typing (MLST), and restriction-modification tests were used to compare the molecular characteristics of methicillin-resistant Staphylococcus aureus (MRSA) isolates recovered at two times within a 10year interval (1998 and 2008) from a tertiary Brazilian hospital. In addition, the antimicrobial susceptibility profiles were analyzed. All 48 MRSA isolates from 1998 and 85.7\% from 2008 (48/56 isolates) displayed multidrug-resistance phenotypes and SCCmec III. All but one of the 13 representative SCCmec III isolates belonged to CC8 and had PFGE patterns similar to that of the BMB9393 strain (Brazilian epidemic clone of MRSA; BEC). In 2008, we found an increased susceptibility to rifampicin and chloramphenicol among the SCCmec III isolates. In addition, we detected the entrance of diverse international MRSA lineages susceptible to trimethoprim-sulfamethoxazole (SXT), almost all belonging to CC5. These non-SCCmec III isolates were related to the USA300 (ST8-SCCmec IV; PFGE-type B), USA800 (ST5-SCCmec IV; subtype D1), USA100 (ST5-SCCmec II; subtype $\mathrm{D}_{2}$ ), and EMRSA-3/Cordobes (ST5-SCCmec I, type C) clones. To the best of our knowledge, this is the first report of the emergence of isolates genetically related to the EMRSA-3/Cordobes clone in southeast Brazil. In this regard, these isolates were the most common non-SCCmec III MRSA in our institution, accounting for $8.9 \%$ of all isolates recovered in 2008 . Thus, despite the supremacy of BEC isolates in our country, significant changes may occur in local MRSA epidemiology, with possible consequences for the rationality of MRSA empiric therapy.
\end{abstract}

Key words: MRSA; Antimicrobial susceptibility; CC5; Cordobes clone; Trimethoprim-sulfamethoxazole

\section{Introduction}

Methicillin-resistant Staphylococcus aureus (MRSA) is a major cause of nosocomial infections worldwide and is a serious source of concern due to the fact that multi-drug resistance is critically important to antimicrobials. The resistance to methicillin and other $\beta$-lactam antibiotics is conferred by the mecA gene, which is inserted into the mobile genetic element staphylococcal chromosomal cassette mec (SCCmec). Currently, eleven major variants of SCCmec (type I to XI) have been characterized (http://www.sccmec. org/Pages/SCC_TypesEN.html. Accessed November 26,
2011). Some SCCmec elements, including types II and III, harbor additional genes that provide resistance to heavy metals and drugs other than $\beta$-lactams, while others, like SCCmec types IV and V, usually carry no additional drug resistance genes other than mecA (1). Epidemiological studies based on SCCmec typing, sequence types (ST) as defined by multilocus sequence typing (MLST), and pulsed-field gel electrophoresis (PFGE) have identified few pandemic hospital-associated MRSA clones, including the Iberian (USA500-related MRSA; ST247-SCCmec I), Brazil-

Correspondence: A.G. Oliveira, Instituto de Ciências Biológicas e Naturais, Universidade Federal do Triângulo Mineiro, Praça Manoel Terra, 330, 38015-050 Uberaba, MG, Brasil. Fax: +55-34-3318-5462; E-mail: agoliveira@dcb.uftm.edu.br

${ }^{*}$ Both are senior authors and have contributed equally.

Received October 3, 2011. Accepted April 9, 2012. Available online April 27, 2012. Published July 2, 2012. 
ian epidemic clone/Hungarian (BEC; ST239-SCCmec III), New York/Japan (USA100-related; ST5-SCCmec II), Paediatric (USA800-related; ST5-SCCmec IV or VI), EMRSA-15 (Barnim epidemic MRSA; ST22-SCCmec IV), EMRSA-16 (USA200-related; ST36-SCCmec II), and Berlin (USA600related; ST45-SCCmec II/IV) (1). MLST analysis, based on sequence variation at seven housekeeping genes, has shown that these pandemic MRSA clones evolved from six different clonal complexes (CC1, CC5, CC8, CC22, CC30, and CC45), each arising from a distinct ancestral genotype $(2,3)$. There is clear evidence that successful methicillinsusceptible $S$. aureus (MSSA) clones have become MRSA by the introduction of SCCmec on more than one occasion. These horizontal transfers of the mec element into different lineages have been highly significant in MRSA evolution (2). However, despite the fact that many SCCmec types, including I, II, and IV, have entered in more than one MSSA background, the SCCmec type III is uniquely associated with the ST239 subgroup of CC8 (4). In fact, ST239 has a mosaic chromosome that seems to have originated from an ST8 ancestral that received a significant part of its genome from an ST30 background (2).

Although each geographical location would only have a limited number of MRSA clones in its hospitals, the geographical distribution of MRSA clones continuously evolves by the introduction of new clones or the evolution of an existing one, usually better adapted and showing enhanced pathogenic properties (5). As a result, the 21st century has been marked by important changes in the epidemiology of MRSA with the emergence of community-associated, non-multidrug-resistant clones as important hospital pathogens, including USA300, and multidrug-resistant clones, traditionally linked to the hospital, as etiological agents of community infections $(6,7)$. Because specific MRSA clones can display different antibiotic resistance profiles, monitoring the distribution and evolution of MRSA is important in controlling bacterial transmission and supporting the use of effective treatments against the most prevalent clones in each local region. In Brazil, despite the high prevalence of MRSA, published data on susceptibility and molecular evolution of MRSA over time are still scarce. Therefore, the aim of this study was to compare the molecular characteristics and the antimicrobial susceptibility profiles of clinical MRSA isolates obtained at two distinct times, within a 10-year interval, in a Brazilian hospital.

\section{Material and Methods}

\section{Study design and identification of isolates}

The study was performed over two time periods with a 10-year interval (1998 and 2008) in the hospital of Universidade Federal do Triângulo Mineiro (UFTM). This hospital, located in Minas Gerais State in Southeastern Brazil, is a 294-bed tertiary-care teaching hospital with a 40-bed intensive care unit (ICU) and a full range of medical specialties.
The $S$. aureus isolates recovered from hospitalized patients were identified by conventional routine methods (Gram staining, catalase and free coagulase tests). Only the first isolate of each patient was considered. All isolates were confirmed as MRSA by PCR amplification of an internal fragment of the mecA gene (8). The present study was approved by the Ethics Committee of Universidade Federal do Triângulo Mineiro.

\section{Susceptibility testing}

The susceptibility profile was determined by the disk diffusion method for the following antimicrobial agents: cefoxitin (CFX), chloramphenicol (CLO), ciprofloxacin (CIP), clindamycin (CLI), erythromycin (ERY), gentamicin (GEN), linezolid (LIN), norfloxacin (NOR), rifampicin (RIF), tetracycline (TET), and trimethoprim-sulfamethoxazole (SXT) (DME, Brazil). The minimal inhibitory concentration (MIC) was determined by the broth dilution method for oxacillin (OXA) and vancomycin (VAN) (Sigma, USA). The disk diffusion test and MIC were performed and interpreted according to guidelines established by the Clinical and Laboratory Standards Institute (9). Inducible macrolide, lincosamide, and streptogramin B resistance $\left(\mathrm{MLS}_{\mathrm{Bi}}\right)$ was tested using the D-test (9). S. aureus ATCC 25923 and S. aureus ATCC 29213 were used for quality control purposes.

\section{Molecular characterization}

$\mathrm{SCC}$ mec typing was performed for all MRSA isolates using a PCR multiplex method (10). The CC was resolved using the restriction-modification (RM) tests according to Cockfield et al. (3). PFGE of the Smal-digested DNA was performed as described previously (11). PFGE-banding patterns were analyzed by visual inspection and interpreted according to the criteria of Tenover et al. (12). Isolates were considered to belong to different types when the banding patterns revealed more than six-band differences. Representative MRSA isolates of BEC (BMB9393) (11) and of USA100, USA300, USA500, and USA800 (kindly provided by Paul Dunman, University of Nebraska Medical Center, USA) were used in the molecular experiments as reference strains. MLST was carried out only for selected isolates previously typed by PFGE and SCCmec that were not related to the reference strains used. The allelic profiles and ST were assigned with reference to the MLST database (http:// www.mlst.net). The lukF gene, coding for the F-subunit of the Panton-Valentine leukocidin (PVL) was assayed by PCR using primers and procedures described by Ribeiro et al. (13). The DNA from the USA300 prototype was used as positive control for the lukF gene.

\section{Statistical analysis}

Data were analyzed with the Epi-Info statistical software (version 3.5.1; CDC, USA). The chi-square or the Fisher exact test was used to compare the antimicrobial susceptibility rates between the two study periods. Differences 
were regarded as significant when $P \leq 0.05$.

\section{Results}

A total of 208 and 151 non-repetitive clinical S. aureus isolates were recovered in 1998 and 2008, respectively, including cases of hospital infections, colonizations and outside-hospital acquisitions. Among them, 48 (23\%) in 1998 and $56(37 \%)$ in 2008 were classified as MRSA according to the results of mecA detection and the phenotypic susceptibility tests performed (Table 1). The MRSA isolates were obtained from the following sites: blood and catheter tips: 21 (1998) and 20 (2008); skin or surgical site: 19 (1998) and 25 (2008); lower respiratory tract secretions: 5 (1998) and 9 (2008); urine: 2 (1998) and 2 (2008); upper respiratory tract secretion: 1 (1998). All the 48 MRSA isolated in 1998 harbored SCCmec III. However, among the 56 isolates recovered in 2008, 48 (85.7\%) harbored SCCmec III and $8(14.3 \%)$ carried diverse types of SCCmec, including SCCmec I ( $\mathrm{N}=5 ; 8.9 \%)$, SCCmec IV $(\mathrm{N}=2 ; 3.6 \%)$ and SCCmec II $(\mathrm{N}=1 ; 1.8 \%)$. The SCCmec IV isolates were tested for the presence of the PVL gene (lukF) but amplification products were not detected.

The antimicrobial susceptibility profiles of the MRSA isolates are summarized in Table 1. During both periods (1998 and 2008), MRSA isolates were highly resistant to ERY, CIP, CLI, NOR, GEN, and TET. However, MRSA isolates recovered in 2008 showed a significant increase in susceptibility to CLO (69.6 vs 4.2\%; P < 0.001 ), RIF (42.9 vs 14.6\%; $\mathrm{P}=0.002$ ) and SXT (14.3 vs $0 \%$; $\mathrm{P}=0.006)$ compared to those recovered in 1998 . A large proportion of MRSA isolates susceptible to CLO (79.5\%; 31/39) and RIF (70.8\%; 17/24) harbored $\mathrm{SCCmec}$ III, while none of the isolates susceptible to SXT carried this SCCmec type. Seven (14.6\%) isolates in 1998 and none in 2008 showed inducible resistance to $\mathrm{CLI}\left(\mathrm{MLS}_{\mathrm{Bi}}\right)$. No MRSA isolate was resistant to VAN and LIN.

Based on the knowledge that SCCmec III has entered only the ST239 background and that BEC isolates predominate in Brazil, only $13 \mathrm{SCCmec} \mathrm{III}$ representative MRSA isolates from 1998 (4 isolates) and 2008 (9 isolates) were analyzed by PFGE and RM tests. Because in 2008 susceptibility to CLO and RIF emerged among the $\mathrm{SCCmec}$ III isolates, a higher number of SCCmec III isolates were selected in 2008 in order to include representatives of these susceptibility profiles. Indeed, because SCCmec I, II and IV can be frequently found in different MRSA backgrounds, all 8 non-SCCmec III isolates detected in this study were analyzed by PFGE and RM tests. These 21 MRSA isolates analyzed showed 5 different PFGE types, namely A to $E$ (Figure $1 A$ and $B$ ). All but one of the $13 \mathrm{SCCmec} \mathrm{III} \mathrm{isolates} \mathrm{analyzed} \mathrm{displayed}$ PFGE pattern A (Figure 1A, subtypes $A_{1-6}$ ) similar to
BMB9393, a prototype strain of BEC. The $13 \mathrm{SCCmec}$ III isolates were confirmed to belong to CC8 by RM tests. One isolate that also displayed SCCmec III and CC8, recovered in 2008, had more than six different bands from BEC and other prototype strains (Figure 1B, type E, lane 10).

Among the remaining isolates recovered in 2008 , one MRSA (isolate 214) displaying SCCmec IV differed from BEC pattern $A_{1}$ by more than six bands and belonged to CC8. This PFGE pattern, named type B (Figure 1B, lane 2), was quite similar to the representative of the USA300 clone. All the $5(8.9 \% ; 5 / 56)$ MRSA isolates carrying $\mathrm{SCCmec}$ I were grouped in the PFGE cluster C (Figure $1 \mathrm{~B}$, subtype $\mathrm{C}_{1}$, lanes 3,4 , and 5 ; subtype $C_{2}$, lanes 6 and 7 ) and belonged to CC5 but differed from the reference strains by more than six PFGE bands. Because this clone was novel in this country, one CC5-SCCmec I MRSA (isolate 119) was selected for MLST analysis and displayed the allelic profile 1-4-1-4-12-1-10, which defines ST5. Two isolates grouped in the PFGE type D also belonged to CC5. Of these, one (isolate 214) harbored SCCmec IV and had a PFGE pattern (Figure $1 \mathrm{~B}$, subtype $\mathrm{D}_{1}$, lane 8 ) similar to that of the USA800/Paediatric clone (ST5-SCCmec IV). The other isolate of cluster $D$ (Figure $1 B$, subtype $D_{2}$, lane 9 ) displayed SCCmec II and was considered to be related to the USA100/New York/Japan clone (ST5-SCCmec II).

The antimicrobial susceptibility profiles and oxacillin MIC of the 21 MRSA isolates analyzed by SCCmec typing, PFGE and RM tests are listed in Table 2. All isolates belonging

Table 1. Antimicrobial susceptibility of the methicillin-resistant Staphylococcus aureus (MRSA) isolates recovered in 1998 and 2008 from a Brazilian tertiary hospital.

\begin{tabular}{lcc}
\hline Antimicrobial agent & \multicolumn{2}{c}{ Number of susceptible isolates (\%) } \\
\cline { 2 - 3 } & $1998(\mathrm{~N}=48)$ & $2008(\mathrm{~N}=56)$ \\
\hline Chloramphenicol & $2(4.2)$ & $39(69.6)^{\mathrm{a} *}$ \\
Ciprofloxacin & $0(0)$ & $1(1.8)$ \\
Clindamycin & $0(0)$ & $2(3.6)$ \\
Erythromycin & $0(0)$ & $0(0)$ \\
Gentamicin & $0(0)$ & $4(7.1)$ \\
Linezolid & $48(100)$ & $56(100)$ \\
Norfloxacin & $0(0)$ & $2(3.6)$ \\
Rifampicin & $7(14.6)$ & $24(42.9)^{\mathrm{b} *}$ \\
Tetracycline & $3(6.3)$ & $6(10.7)$ \\
Trimethoprim-sulfamethoxazole & $0(0)$ & $8(14.3)^{\mathrm{c} *}$ \\
Vancomycin & $48(100)$ & $56(100)$ \\
\hline
\end{tabular}

aThirty-one (79.5\%) of the 39 and ${ }^{\mathrm{b}} 17$ (70.8\%) of the 24 MRSA isolates susceptible to chloramphenicol and rifampicin, respectively, carried $\mathrm{SCC} m e c$ III. CNone of the $8 \mathrm{MRSA}$ isolates susceptible to trimethoprimsulfamethoxazole carried SCCmec III. SCCmec III = staphylococcal chromosomal cassette mec III; MRSA = methicillin-resistant Staphylococcus aureus. ${ }^{*} \mathrm{P}<0.05$ compared to 1998 (chi-square or Fisher exact test). 

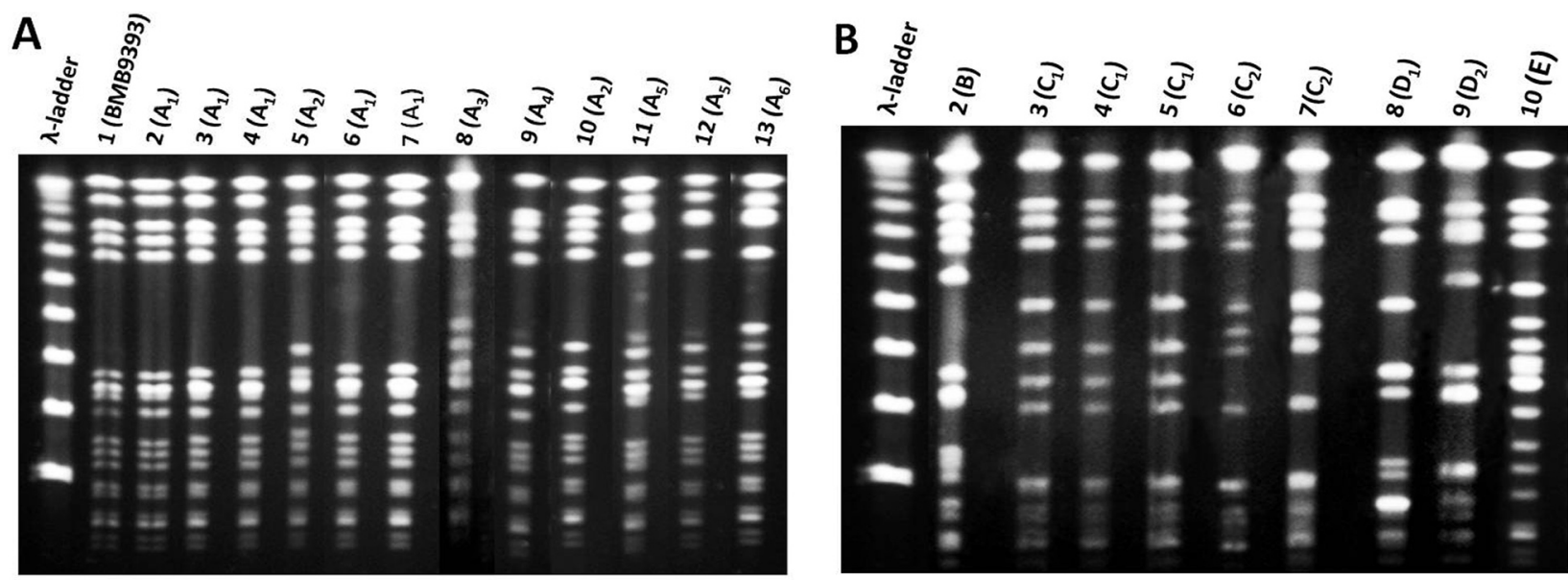

Figure 1. Pulsed-field gel electrophoresis (PFGE) patterns of Smal-digested genomic DNA of MRSA isolates recovered in 1998 and 2008 from a Brazilian tertiary hospital. A, MRSA isolates displaying patterns quite similar to that of BEC (BMB9393). Lane 1, DNA molecular size marker ( $\lambda$-ladder, 50-1000 kb); lane 2, strain BMB9393, representative of the BEC; lanes 3-5, pattern $A_{1}$ (isolates 1, 2, and 15; 1998); lane 6, pattern $A_{2}$ (isolate 19; 1998); lanes 7 and 8 , pattern $A_{1}$ (isolates 12 and 15; 2008), lane 9, pattern $A_{3}$ (isolate 2; 2008); lane 10, pattern $A_{4}$ (isolate 42; 2008); lane 11, pattern $A_{2}$ (isolate 53; 2008); lanes 12 and 13, pattern $A_{5}$ (isolates 8 and 40; 2008); lane 14, pattern $A_{6}$ (isolate 50; 2008). $B$, MRSA isolates displaying patterns different from those of BEC. Lane 1, DNA molecular size marker ( $\lambda$-ladder, $50-1000 \mathrm{~kb}$ ); lane 2, pattern $\mathrm{B}$ (isolate 214; 2008); lanes 3-5, pattern $\mathrm{C}_{1}$ (isolates 3, 119, 251; 2008); lanes 6 and 7, pattern $C_{2}$ (134 and 140; 2008); lanes 8 and 9, pattern $D_{1}$ and $D_{2}$ (isolates 174 and 190; 2008); lane 10, pattern $E$ (isolate 9 ; 2008). $\mathrm{BEC}=$ Brazilian epidemic clone of MRSA; MRSA = methicillin-resistant Staphylococcus aureus.

to CC5 displayed SCCmec I, II or IV and were susceptible to SXT, while only one CC8 isolate, harboring SCCmec IV (USA300-related), showed susceptibility to this drug.

\section{Discussion}

The use of a variety of molecular typing techniques allowed the detection of MRSA clones circulating in our hospital at different time periods. In this study, in 1998, multidrug-resistant isolates harboring SCCmec III were the only MRSA detected in the hospital, whereas in the latter study period (2008) new epidemic MRSA clones emerged, accounting for $14.3 \%$ of the MRSA isolates recovered during this period. It is noteworthy that all non-SCCmec III isolates found in 2008 were characteristically susceptible to SXT and all but one belonged to CC5.

Actually, SCCmec III is uniquely associated with the widespread ST239-related MRSA (4) and ST239-III MRSA is an universally occurring multidrug-resistant lineage, first described in 1992 in Brazil (11), responsible for an incredible number of hospital-acquired infections in several countries on diverse continents (1). However, ST239 isolates have been replaced by other MRSA backgrounds in some countries. In Portugal, from 1996 to 2000, BEC isolates were the most common ST239 MRSA inside hospitals, whereas, since 2001 , this clone has drastically declined in that country with a concomitant increase of isolates belonging to EMRSA-15 (ST22-SCCmec IV) (14). In Argentina, Chile and Paraguay, the BEC was displaced by the Cordobes clone
(ST5-SCCmec I) (15). More recently, a study conducted in Rio de Janeiro, Brazil, showed that isolates classified as ST1-SCCmec IV (USA400-related) were replacing BEC in the two hospitals evaluated (16).

Besides the SCCmec III isolates, the only CC8 MRSA found in our study was related to USA300 (ST8-SCCmec IV), which has been mostly associated with community acquired (CA)-MRSA infections $(1,6)$. Usually, USA300related isolates have been linked to the production of $P V L$ and susceptibility to almost all classes of non- $\beta$-lactamic antimicrobials (1). Despite the fact that the PVL-encoding gene lukF was not detected in the USA300-related isolate found in our hospital, this isolate was recovered from a patient with orbital cellulitis classified as a CA infection, according to CDC guidelines. In Brazil, infections associated with PVL-negative USA300 isolates have already been reported $(13,17,18)$.

All other non-SCCmec III isolates recovered in 2008 were allocated to CC5. In contrast to ST239, MRSA isolates belonging to ST5 (CC5) have been associated with different SCCmec types (i.e., I, II, and IV) comprising a large number of MRSA clones, both hospital acquired (HA)- and CA-MRSA, some of which have attained pandemic spread (2). Most of the CC5 isolates found in the current study harbored SCCmec I and clustered in the same PFGE type. MLST analysis of one of these isolates showed that it belonged to ST5. Four isolates were associated with cases of colonization and one (isolate 3 ) with a case of HA pneumonia in an ICU patient. 
Table 2. Molecular characteristics, minimal inhibitory concentration (MIC) for oxacillin and antimicrobial susceptibility profile of 21 representative MRSA isolates recovered in 1998 and 2008 from a Brazilian tertiary hospital.

\begin{tabular}{|c|c|c|c|c|c|c|c|}
\hline Year of isolation & Isolate & Pulsotype & SCCmec type & $\mathrm{CC}$ & OXA MIC & Antimicrobial susceptibility & PFGE clone \\
\hline \multirow[t]{4}{*}{1998} & 1 & A1 & III & $\mathrm{CC} 8$ & 256 & LIN, VAN & BEC \\
\hline & 2 & $\mathrm{~A} 1$ & III & $\mathrm{CC} 8$ & 256 & LIN, VAN & BEC \\
\hline & 15 & A1 & III & $\mathrm{CC} 8$ & $>256$ & LIN, VAN & BEC \\
\hline & 19 & $\mathrm{~A} 2$ & III & $\mathrm{CC} 8$ & 256 & LIN, VAN & BEC \\
\hline \multirow[t]{17}{*}{2008} & 12 & $\mathrm{~A} 1$ & III & $\mathrm{cc} 8$ & 256 & LIN, RIF, VAN & BEC \\
\hline & 15 & $\mathrm{~A} 1$ & III & $\mathrm{CC} 8$ & $>256$ & CLO, LIN, RIF, VAN & BEC \\
\hline & 2 & A3 & III & $\mathrm{CC} 8$ & 256 & CLO, LIN, RIF, VAN & BEC \\
\hline & 42 & A4 & III & $\mathrm{CC} 8$ & 256 & LIN, VAN & BEC \\
\hline & 53 & $\mathrm{~A} 2$ & III & $\mathrm{CC} 8$ & $>256$ & LIN, VAN & BEC \\
\hline & 8 & A5 & III & $\mathrm{CC} 8$ & 256 & CLO, LIN, RIF, TET, VAN & BEC \\
\hline & 40 & A5 & III & $\mathrm{CC} 8$ & 128 & LIN, RIF, VAN & BEC \\
\hline & 50 & A6 & III & $\mathrm{CC} 8$ & $>256$ & LIN, VAN & BEC \\
\hline & 214 & B & IV & $\mathrm{CC} 8$ & 16 & $\begin{array}{l}\text { CLO, CLI, GEN, LIN, RIF, } \\
\text { SXT, TET, VAN }\end{array}$ & USA300 \\
\hline & 3 & $\mathrm{C} 1$ & I & CC5 & 256 & CLO, LIN, RIF, SXT, VAN & EMRSA-3/Cordobes \\
\hline & 119 & $\mathrm{C} 1$ & I & CC5 & 256 & CLO, LIN, RIF, SXT, VAN & EMRSA-3/Cordobes \\
\hline & 251 & $\mathrm{C} 1$ & I & $\mathrm{cc} 5$ & 256 & $\begin{array}{l}\text { CLO, LIN, NOR, RIF, } \\
\text { SXT, TET, VAN }\end{array}$ & EMRSA-3/Cordobes \\
\hline & 134 & $\mathrm{C} 2$ & I & CC5 & $>256$ & CLO, LIN, RIF, SXT, VAN & EMRSA-3/Cordobes \\
\hline & 140 & $\mathrm{C} 2$ & I & CC5 & $>256$ & CLO, LIN, SXT, VAN & EMRSA-3/Cordobes \\
\hline & 174 & D1 & IV & CC5 & 16 & $\begin{array}{l}\text { CLO, CLI, GEN, LIN, } \\
\text { NOR, RIF, SXT, TET, VAN }\end{array}$ & PC (USA800) \\
\hline & 190 & $\mathrm{D} 2$ & II & CC5 & 32 & $\begin{array}{l}\text { CLO, GEN, LIN, RIF, SXT, } \\
\text { TET, VAN }\end{array}$ & NY/J (USA100) \\
\hline & 9 & $\mathrm{E}$ & III & $\mathrm{CC} 8$ & 256 & CLO, LIN, RIF, VAN & Undetermined \\
\hline
\end{tabular}

MRSA = methicillin-resistant Staphylococcus aureus; $\mathrm{SCCmec}=$ staphylococcal chromosomal cassette mec; $\mathrm{CC}=$ clonal complex; PFGE = pulsed-field gel electrophoresis; CIP = ciprofloxacin; CLI = clindamycin; CLO = chloramphenicol; GEN = gentamicin; LIN = linezolid; OXA = oxacillin; RIF = rifampicin; SXT = trimethoprim-sulfamethoxazole; TET = tetracycline; VAN = vancomycin; $\mathrm{BEC}=$ Brazilian epidemic clone; $\mathrm{PC}=$ pediatric clone; $\mathrm{NY} / \mathrm{J}=\mathrm{New}$ York/Japan clone.

The genotype ST5-SCCmecl is shared by the EMRSA-3 and Cordobes clones. The EMRSA-3 clone, detected in the United Kingdom in 1987-1988, is among the most common MRSA in that region along with EMRSA-15 and EMRSA-16 (2). Studies conducted in Chile (1997 and 1998) and Argentina (1999) have identified the Cordobes clone, which is genetically related to the EMRSA-3 but presents differences in the PFGE pattern and spa type $(15,19,20)$. It is of note that in hospitals of these countries as well as in Paraguay, the ST239 isolate was replaced by the multidrug-resistant Cordobes clone (15). To our knowledge, isolates related to the EMRSA-3 and Cordobes clones have not been reported in southeast Brazil thus far. After our study was concluded, this clone was reported in Porto Alegre, Rio Grande do Sul State (21), which is the southernmost state in Brazil located on the border with Argentina.

Two other CC5 MRSA clones, related to the USA800/
Paediatric clone (SCCmec IV) and USA100/New York/Japan (SCCmec II), were sporadically detected in our hospital. MRSA isolates belonging to both of these international clones, which are commonly spread in nosocomial settings, had been previously described in Brazil $(16,22,23)$. In other Latin America countries, the USA800/Paediatric and USA100/New York/Japan CC5 clones have successfully spread along with the Cordobes clone (15).

During both periods of the study (1998 and 2008), MRSA isolates were highly resistant to ERY, CIP, CLI, NOR, GEN, and TET although all of them were susceptible to VAN and LIN. During the last study period (2008), we detected a great proportion of SCCmec III isolates susceptible to CLO and RIF compared to those observed 10 years earlier. Although we did not test this hypothesis, these increased susceptibilities may possibly be an indication of the entry of a novel ST239-SCCmec III variant in our hospital. In a 
Chinese hospital, for instance, it was demonstrated that the ST239-spa t037 clone, displaying increased susceptibility to RIF, was replaced by the ST239-spa t030 clone, frequently more resistant to this drug (24). After 2000, the prevalence of MRSA rapidly increased in that hospital, which was coincident with the emergence of the ST239-spa t030 clone that, according to the authors, seemed to have a stronger survival advantage and could easily be transmitted. In that study, ST239 was also the most common lineage over time (24). In fact, in previous studies, we had found that some ST239-SCCmec III variants might display an increased ability to accumulate biofilm and to invade and adhere to human airway epithelial cells (5).

The increased susceptibility to SXT detected in 2008 was due to the emergence of CC5 (SCCmec I, II and IV) isolates since all of them were susceptible to this drug. In contrast, SCCmec III isolates were typically resistant to SXT, as shown in our study and others $(14,16)$. Therefore, the analysis of the antimicrobial susceptibility profile may be useful as a phenotypic marker for a preliminary search for new MRSA clones in hospitals where ST239-SCCmec III-related isolates predominate. Moreover, SXT might offer an additional therapeutic option to clinicians for the treatment of infections caused by the more susceptible MRSA isolates because it is inexpensive, bactericidal, has an acceptable toxicity profile, penetrates well into tissue and is available for administration by both the oral and intrave-

\section{References}

1. Deurenberg RH, Vink C, Kalenic S, Friedrich AW, Bruggeman CA, Stobberingh EE. The molecular evolution of methicillin-resistant Staphylococcus aureus. Clin Microbiol Infect 2007; 13: 222-235.

2. Enright MC, Robinson DA, Randle G, Feil EJ, Grundmann $H$, Spratt BG. The evolutionary history of methicillin-resistant Staphylococcus aureus (MRSA). Proc Natl Acad Sci U S A 2002; 99: 7687-7692.

3. Cockfield JD, Pathak S, Edgeworth JD, Lindsay JA. Rapid determination of hospital-acquired meticillin-resistant Staphylococcus aureus lineages. J Med Microbiol 2007; 56: 614-619.

4. Chen L, Mediavilla JR, Smyth DS, Chavda KD, Ionescu $\mathrm{R}$, Roberts RB, et al. Identification of a novel transposon (Tn6072) and a truncated staphylococcal cassette chromosome mec element in methicillin-resistant Staphylococcus aureus ST239. Antimicrob Agents Chemother 2010; 54: 3347-3354.

5. Amaral MM, Coelho LR, Flores RP, Souza RR, SilvaCarvalho MC, Teixeira LA, et al. The predominant variant of the Brazilian epidemic clonal complex of methicillin-resistant Staphylococcus aureus has an enhanced ability to produce biofilm and to adhere to and invade airway epithelial cells. $J$ Infect Dis 2005; 192: 801-810.

6. Limbago B, Fosheim GE, Schoonover V, Crane CE, Nadle $\mathrm{J}$, Petit $\mathrm{S}$, et al. Characterization of methicillin-resistant nous routes. However, controlled clinical trials describing successful therapy with SXT for MRSA infections compared to VAN and other available anti-MRSA drugs are still very scarce (25).

Despite the predominance of SCCmec III MRSA isolates in our hospital, changes in the clonal nature were detected in 2008 represented by the entry of diverse MRSA lineages, almost all belonging to CC5. In addition, our data suggest that SXT susceptibility may be used as an antimicrobial marker to indicate the potential emergence of new MRSA clones, mainly in hospitals where ST239 isolates predominate. The emergence of MRSA isolates genetically related to the EMRSA-3/Cordobes clone in southeast Brazil indicates that the spread of the Cordobes clone in our country is not limited to the Argentinean borders. Finally, these data show that local studies on the molecular epidemiology of MRSA are essential to trace the entry of new clones and to collect information on antimicrobial susceptibility to guide decisions regarding the choice of appropriate empirical therapy for invasive MRSA infections.

\section{Acknowledgments}

Research supported in part by CNPq, FAPERJ, CAPES to A.M.S. Figueiredo, and Fundação de Ensino e Pesquisa de Uberaba (FUNEPU) to A.G. Oliveira.
Staphylococcus aureus isolates collected in 2005 and 2006 from patients with invasive disease: a population-based analysis. J Clin Microbiol 2009; 47: 1344-1351.

7. Rozenbaum R, Silva-Carvalho MC, Souza RR, Melo MC, Gobbi CN, Coelho LR, et al. Molecular characterization of methicillin-resistant Staphylococcus aureus disseminated in a home care system. Infect Control Hosp Epidemiol 2006; 27: 1041-1050.

8. Oliveira DC, de Lencastre H. Multiplex PCR strategy for rapid identification of structural types and variants of the mec element in methicillin-resistant Staphylococcus aureus. Antimicrob Agents Chemother 2002; 46: 2155-2161.

9. CLSI. Clinical and Laboratory Standards Institute. Performance standards for antimicrobial susceptibility testing. Twentieth information supplement. Wayne: CLSI document M100-S20; 2010.

10. Boye K, Bartels MD, Andersen IS, Moller JA, Westh H. A new multiplex PCR for easy screening of methicillin-resistant Staphylococcus aureus SCCmec types I-V. Clin Microbiol Infect 2007; 13: 725-727.

11. Teixeira LA, Resende CA, Ormonde LR, Rosenbaum R, Figueiredo AM, de Lencastre $\mathrm{H}$, et al. Geographic spread of epidemic multiresistant Staphylococcus aureus clone in Brazil. J Clin Microbiol 1995; 33: 2400-2404.

12. Tenover FC, Arbeit RD, Goering RV, Mickelsen PA, Murray $\mathrm{BE}$, Persing $\mathrm{DH}$, et al. Interpreting chromosomal DNA 
restriction patterns produced by pulsed-field gel electrophoresis: criteria for bacterial strain typing. J Clin Microbiol 1995; 33: 2233-2239.

13. Ribeiro A, Dias C, Silva-Carvalho MC, Berquo L, Ferreira FA, Santos RN, et al. First report of infection with community-acquired methicillin-resistant Staphylococcus aureus in South America. J Clin Microbiol 2005; 43: 1985-1988.

14. Amorim ML, Faria NA, Oliveira DC, Vasconcelos C, Cabeda JC, Mendes AC, et al. Changes in the clonal nature and antibiotic resistance profiles of methicillin-resistant Staphylococcus aureus isolates associated with spread of the EMRSA-15 clone in a tertiary care Portuguese hospital. J Clin Microbiol 2007; 45: 2881-2888.

15. Rodriguez-Noriega E, Seas C, Guzman-Blanco M, Mejia C, Alvarez C, Bavestrello L, et al. Evolution of methicillinresistant Staphylococcus aureus clones in Latin America. Int J Infect Dis 2010; 14: e560-e566.

16. Silva-Carvalho MC, Bonelli RR, Souza RR, Moreira S, dos Santos LC, de Souza CM, et al. Emergence of multiresistant variants of the community-acquired methicillin-resistant Staphylococcus aureus lineage ST1-SCCmecIV in 2 hospitals in Rio de Janeiro, Brazil. Diagn Microbiol Infect Dis 2009; 65: 300-305.

17. Carmo MS, Inoue F, Andrade SS, Paschoal L, Silva FM, Oliveira VG, et al. New multilocus sequence typing of MRSA in São Paulo, Brazil. Braz J Med Biol Res 2011; 44: 10131017.

18. Schuenck RP, Nouer SA, Winter CO, Cavalcante FS, Scotti TD, Ferreira AL, et al. Polyclonal presence of non-multiresistant methicillin-resistant Staphylococcus aureus isolates carrying SCCmec IV in health care-associated infections in a hospital in Rio de Janeiro, Brazil. Diagn Microbiol Infect
Dis 2009; 64: 434-441.

19. Aires DeSousa M, Miragaia M, Sanches IS, Avila S, Adamson I, Casagrande ST, et al. Three-year assessment of methicillin-resistant Staphylococcus aureus clones in Latin America from 1996 to 1998. J Clin Microbiol 2001; 39: 21972205.

20. Sola C, Cortes P, Saka HA, Vindel A, Bocco JL. Evolution and molecular characterization of methicillin-resistant Staphylococcus aureus epidemic and sporadic clones in Cordoba, Argentina. J Clin Microbiol 2006; 44: 192-200.

21. Becker AP, Santos O, Castrucci FM, Dias C, D'Azevedo PA. First report of methicillin-resistant Staphylococcus aureus Cordobes/Chilean clone involved in nosocomial infections in Brazil. Epidemiol Infect 2011; 1-4.

22. Scribel LV, Silva-Carvalho MC, Souza RR, Superti SV, Kvitko $\mathrm{CH}$, Figueiredo AM, et al. Clinical and molecular epidemiology of methicillin-resistant Staphylococcus aureus carrying SCCmeclV in a university hospital in Porto Alegre, Brazil. Diagn Microbiol Infect Dis 2009; 65: 457-461.

23. de Miranda OP, Silva-Carvalho MC, Ribeiro A, Portela F, Cordeiro RP, Caetano N, et al. Emergence in Brazil of methicillin-resistant Staphylococcus aureus isolates carrying SCCmecIV that are related genetically to the USA800 clone. Clin Microbiol Infect 2007; 13: 1165-1172.

24. Chen $\mathrm{H}$, Liu $Y$, Jiang $X$, Chen $M$, Wang $H$. Rapid change of methicillin-resistant Staphylococcus aureus clones in a Chinese tertiary care hospital over a 15 -year period. Antimicrob Agents Chemother 2010; 54: 1842-1847.

25. Proctor RA. Role of folate antagonists in the treatment of methicillin-resistant Staphylococcus aureus infection. Clin Infect Dis 2008; 46: 584-593. 